\title{
Electro-optical target system for position and speed measurement
}

\author{
S.-T. Lu \\ C. Chou \\ M.-C. Lee \\ Y.-P. Wu
}

Indexing terms: External ballistics, Noncontact measurement, Electro-optical techniques, Position measurement, Speed measurement

Abstract: This paper introduces an electro-optical target system (EOTS) covering the speed range from subsonic to supersonic. This microcomputerbased system has a novel structure and shows the capability of precisely detecting the position as well as the velocity of small calibre projectiles in real time. A prototype EOTS whose target area is $1 \mathrm{~m}^{2}$ has been constructed and tested. A speed accuracy of better than $0.3 \%$ was achieved. A position accuracy, mainly dependent on the spacing between photodiodes in EOTS, of better than $1 \mathrm{~mm}$ on a target area of $1 \mathrm{~m}^{2}$ was also accomplished.

\section{Introduction}

The speed and position measurements of projectiles are two important items in ballistic research. To determine these parameters precisely one needs an accurate measuring system. A conventional method, namely the hanging up (and taking down) of target discs [1], though accurate in position measuring, is time consuming. A shot-position indicator (SPI), described in Reference 2, can measure the position of a high speed projectile by acoustic measurement. However, the SPI does not provide the speed information; neither does the conventional method. Besides, the SPI is used within the limits of supersonic projectiles.

To measure the speed and position of projectiles rapidly and simultaneously, different electro-optical based systems have been proposed [3-5]. These systems have the ability to cover the speed range from subsonic to supersonic. One system, called the target measurement system (TMS) [3], uses vertical and horizontal banks of light sources to form two perpendicular light grids that construct the target area. Another system, called the electro-optical projectile analyser [4], uses the same principle as TMS, but simplifies light sources with fibre optics bundles and a single light source in each light grid. The other system, called the electronic yaw screen (EYS) [5], uses a solid state laser that is collimated and directed to a one-dimensional beam expander system to form a fan-

(C) IEE, 1993

Paper 9049A (S4), first received 6th January and in revised form 8 th June 1992

C. Chou is with the School of Medical Technology, National Yang Ming Medical College, Taipei, Taiwan, Republic of China

S.-T. Lu, M.-C. Lee and Y.-P. Wu is with the Department of Electrical Engineering, National Taiwan University, Taipei, Taiwan, Republic of China

252 shaped light screen. This light screen then is reflected by a mirror to construct a portion of the target area. The light screen is more precise than the light grid because there is no dead zone in the target area as with the light grid system.

From the aspect of speed and position measurement, we take advantage of the above systems and propose a novel system; the electro-optical target system (EOTS) [6]. We use a cylindrical mirror that reflects the incident laser beam into a $90^{\circ}$ fan-shaped light screen. Two such light screens construct a two-dimensional positioning system. We even propose a bent cylindrical mirror to generate a $90^{\circ}$ light screen with a few degrees extended in a direction normal to the light screen to reduce the sensitivity to vibrations.

A prototype EOTS, whose target area is $1 \mathrm{~m}^{2}$ and measured speed range is from $50 \mathrm{~m} / \mathrm{s}$ to $1200 \mathrm{~m} / \mathrm{s}$, has been constructed and tested. A speed range of up to $5000 \mathrm{~m} / \mathrm{s}$ can also be expected according to the simulation results from the electronic circuit using PSpice [7]. Finally, a nine-point testing result from a 0.38 in. pistol is shown in this paper. The result shows that the standard deviation of position accuracy is less than $1 \mathrm{~mm}$.

\section{Basic principle of EOTS}

Fig. 1 shows the optical configuration of EOTS. A laser beam from a $\mathrm{He}-\mathrm{Ne}$ laser is directed onto a cylindrical mirror. The reflected laser beams create a fan-shaped light screen and are directed onto photodiodes that are neatly arranged into an L-shaped photodiode array. EOTS uses two laser sources, two cylindrical mirrors and two photodiode arrays, which are arranged on the opposite sides of the EOTS body to form two fan-shaped light screens. Each light screen is combined with its own signal processing circuit to construct an optical gate. Although there is a distance between the two parallel light screens, viewed from a distance point, these fan beams intersect in a region of space called the target area (Fig. 2). A projectile can be measured only if it travels through this target area.

Fig. 3 shows the shot position of the projectile is calculated. The target area, for the convenience of illustration, is a square of dimension $D$ on each side. The number of photodiodes on the L-shaped photodiode array is $2 N$. Each photodiode is numbered in order, as shown in the figure. For illustration clarity, only the photodiode array and the cylindrical mirror of the first optical gate are shown. The projectile is considered to be incident normally to the first and to the second optical gate in sequence. When the projectile blocks the light screens, the respective photodiodes will be activated by 
the disturbance. In the first optical gate, the laser beam from the cylindrical mirror to each photodiode makes a unique angle with the $y$-axis. This angle is measured

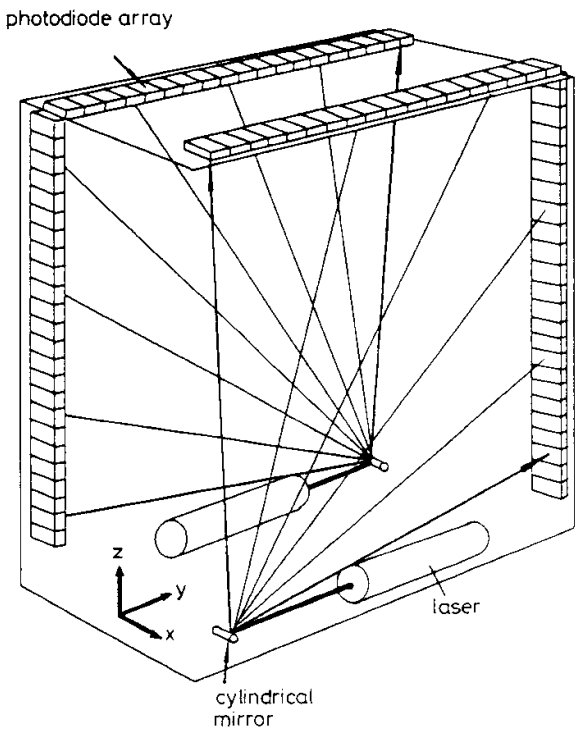

Fig. 1 Optical configuration of EOTS

counter-clockwise from the axis. The angle with respect to a photodiode, numbered $n$, can be calculted as

$$
\delta_{1}(n)=\arctan \frac{\left(n-\frac{1}{2}\right)}{N} \text { for } 1 \leqslant n \leqslant N
$$

and

$$
\delta_{1}(n)=\arctan \frac{N}{\left(2 N-n+\frac{1}{2}\right)} \text { for } N+1 \leqslant n \leqslant 2 N
$$

If certain photodiodes, numbered from $j$ to $k$, are activat ed by projectiles, then the shot-position angle $\delta_{1}$ is given by

$$
\delta_{1}=\frac{\delta_{1}(j)+\delta_{1}(k)}{2}
$$

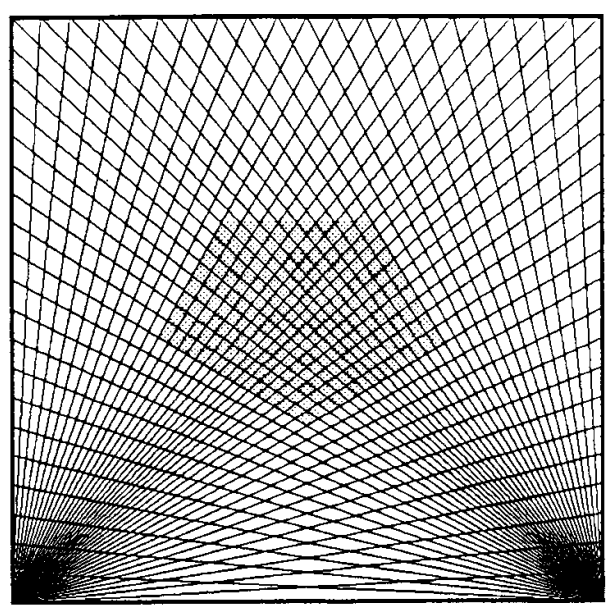

Fig. 2 Intersections of the two light screens in the target area IEE PROCEEDINGS-A, Vol. 140, No. 4, JULY 1993
Similarly, the shot-position angle of the second optical gate $\delta_{2}$, measured clockwise from the minus $y$-axis, is decided. After the two angles have been measured, the

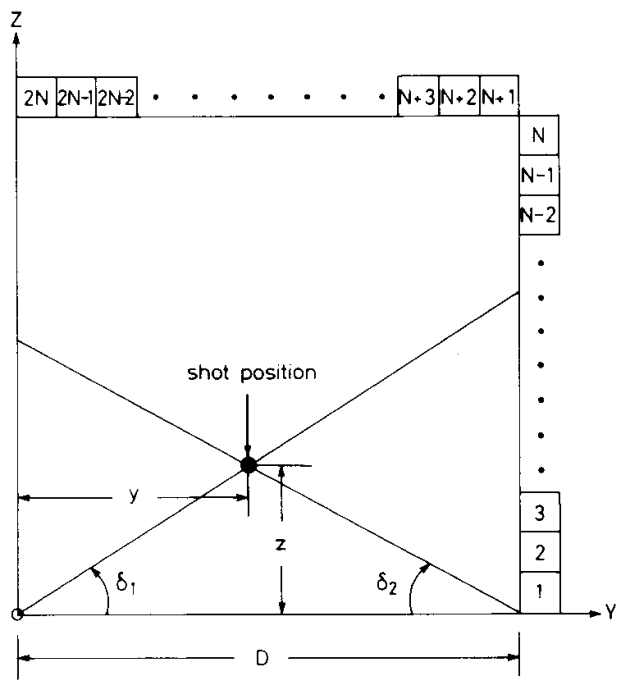

Fig. 3 Illustration of shot-position calculation

shot position of the projectile is deduced in Cartesian coordinates as

$$
y=\frac{D \tan \delta_{2}}{\tan \delta_{1}+\tan \delta_{2}}
$$

and

$$
z=y \tan \delta_{1}
$$

If $S$ is the distance between the two light screens, then the average speed $v$ for the projectile passing through the distance $S$ is given by

$$
v=\frac{S}{T}
$$

where $T$ is the time interval for the projectile to pass through distance $S$.

\section{Configuration of EOTS}

\subsection{Optical system of EOTS}

We use a $\mathrm{He}-\mathrm{Ne}$ laser directed onto a cylindrical mirror to create a light screen. The relation among the laser beam diameter $d$, the cylindrical mirror diameter $w$ and the beam expanding angle $\phi$ is shown in Fig. 4. This relation can be calculated as

$$
\phi=2 \arcsin \frac{2 d}{w}
$$

To create a light screen of which $\phi$ equals $90^{\circ}$, the ratio of $w$ to $d$ is 2.8. Because the $\mathrm{He}-\mathrm{Ne}$ laser beam has Gaussian distribution and each photodiode on the photodiode array has a different distance to the cylindrical mirror, the received laser power at each photodiode is not constant. This will influence the speed accuracy of EOTS (see Fig. 6 and Section 4.1).

\subsection{Analogue circuitry}

EOTs has $2 N$ analogue channels in each of its two optical gates. Every analogue channel has the same struc- 
ture. Each analogue channel contains a photodiode, a linear amplifier, a band-pass filter and a comparator. The linear amplifier amplifies the signal coming from the photodiode. The band-pass filter filters noises such as

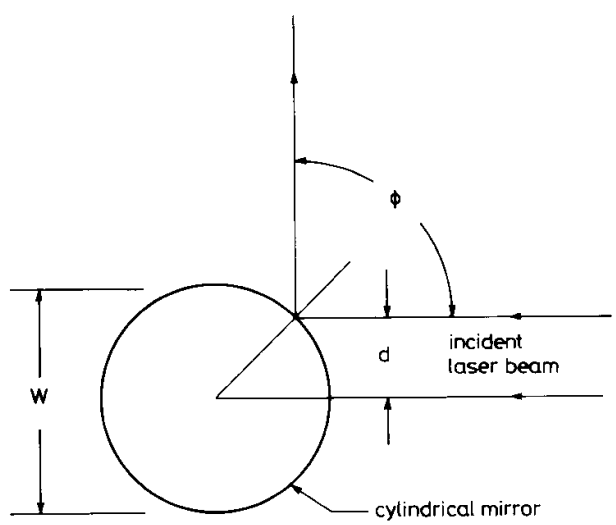

Fig. 4 Laser beam directs on a cylindrical mirror

bugs flying through the light screen and flicker of other light sources nearby. The comparator compares the output $V_{o}$ coming from the filter with a threshold voltage $V_{T H}$. If $V_{o}$ is higher than $V_{T H}$, then the comparator will activate a flip-flop (FF) to change the state.

\subsection{Digital circuitry}

Fig. 5 is the block diagram of the digital signal processing circuit. Input coming from the analogue channel is fed to a relative FF. When the projectile blocks the light screen of the first optical gate, the state-changed FFs will make the output of the NAND gate U1 change state. The U1 locks all FFs of the first optical gate to protect genuine projectile data from the influence of shock waves behind the projectile, and starts the counter U5 that operates at a clock frequency of $10 \mathrm{MHz}$. As the projectile blocks the

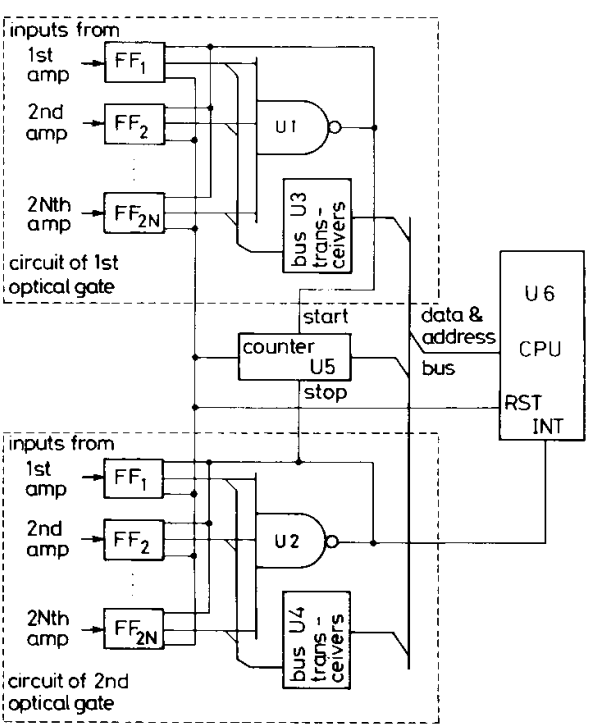

Fig. 5 Block diagram of digital signal processing circuit light screen of the second optical gate, the circuit of the second optical gate functions as the circuit of the first optical gate did, but stops the counter. Moreover, the NAND gate U2 passes an interrupt signal (INT) to the central processing unit (CPU) while U5 is being stopped. The CPU then recognises the interrupt request, picks the projectile data up, and resets U5 and all FFs for the next shot, in sequence. In Fig. 5, the counter relates the time interval $T$ in eqn. 6 . Besides, every photodiode is assigned a specific FF and every $F F$ is given a relative address. Therefore, the CPU will be able to identify which photodiode generates the signal, to decide the impact position of eqns. $1-5$, and to calculate the speed of the projectile.

\section{Accuracy of EOTS}

4.1 Accuracy of speed measurement

The accuracy of projectile velocity measurement with sky-screens has been deduced by Hartwig [8] as

$$
\left|\frac{\Delta v}{v}\right| \leqslant \frac{|\Delta S|+v|\Delta T|}{S}
$$

where parameters were the same as eqn. 6 used. $\Delta v, \Delta S$ and $\Delta T$ are values of maximum error in $v, S$ and $T$, respectively. In EOTS, photodiodes are directed by nonuniform optical power, as described in Section 3.1, which implies that different analogue channels will have different response times, as though they are activated in the same way. Fig. 6 describes the typical input and output waveforms of an analogue channel when a projectile passes through the light screen. The dotted line is the

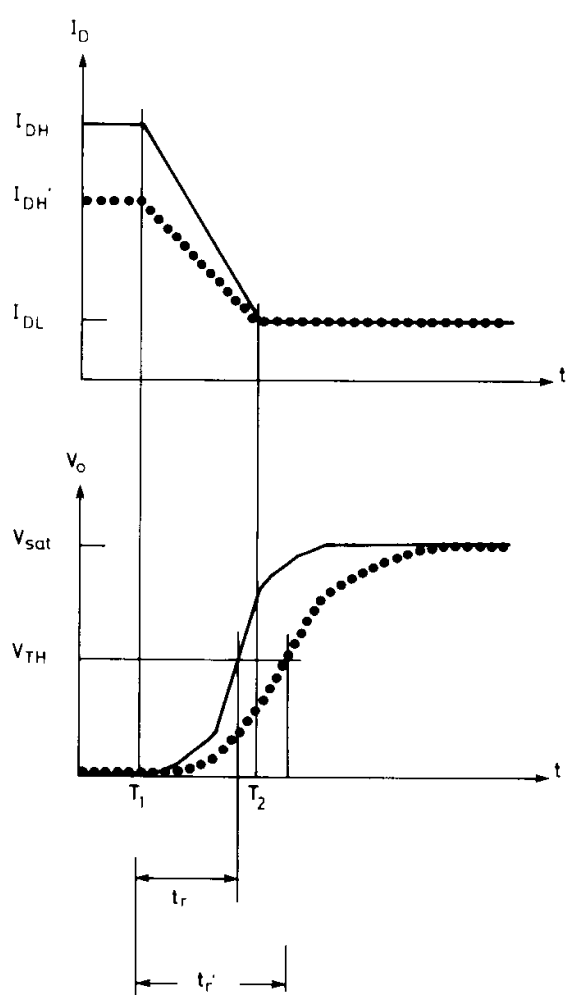

Fig. 6 Typical input and output waveform of analogue channel

IEE PROCEEDINGS-A, Vol. 140, No. 4, JULY 1993 
response of the weaker optical input with respect to the solid line. In this Figure, the optical power density directed onto the photodiode is considered to be constan along the $x$-axis. Referring to the solid line, the projectile touches the light screen at $T_{1}$ and entirely blocks laser beams at $T_{2}$; the activated photodiode current $I_{D}$ drops from $I_{D H}$ to $I_{D L}$. The output voltage $V_{o}$ of the analogue channel then rises to a saturation voltage $V_{\text {sat }}$. The counter is not triggered until $V_{o}$ is larger than $V_{T H}$. The interval from $T_{1}$ to the time that $V_{o}$ equals $V_{T H}$ is called the response time $t_{r}$. From Fig. 6, we can realise that a different input power variation with time will produce a different output response time $t_{r}$. Therefore, the $\Delta T$ of eqn. 8 should include $\Delta t_{r}$ for EOTS, where $\Delta t_{r}$ is the worst-case difference, i.e., the largest $t_{r}$ of the first optical gate minus the smallest $t_{r}$ of the second optical gate Table 1 lists the simulation results of $\Delta t_{r}$ of the analogue circuit with respect to different projectile velocities using PSpice.

Table 1 : Simulation results of $\Delta t_{r}$ respect to projectile speed

\begin{tabular}{cl}
\hline $\begin{array}{l}\text { Projectile speed } \\
\mathrm{m} / \mathrm{s}\end{array}$ & $\begin{array}{l}\Delta t_{r} \\
\mu \mathrm{s}\end{array}$ \\
\hline 200 & 2.13 \\
1000 & 0.44 \\
5000 & 0.08 \\
\hline
\end{tabular}

\subsection{Accuracy of position measurement}

Considering an EOTs structure in Fig. 3, if a photodiode, numbered $n$, is activated by a projectile, the exact shotposition angle $\delta_{e}$ will be within the range

$$
\arctan \left(\frac{n-1}{N}\right) \leqslant \delta_{e} \leqslant \arctan \left(\frac{n}{N}\right) \text { for } 1 \leqslant n \leqslant \mathrm{~N}
$$

or

$$
\arctan \left(\frac{N}{2 N-n+1}\right) \leqslant \delta_{e} \leqslant \arctan \left(\frac{N}{2 N-n}\right)
$$

for $N+1 \leqslant n \leqslant 2 N \quad(10)$

Referring to eqns. 1 and 2, eqns. 9 and 10 express that the worst-case deviation of $\delta, \Delta \delta$ is caused by halfphotodiode-spacing shift of measuring ambiguity. Thus, the deviations of the $y$-axis and $z$-axis can be deduced as

$$
\Delta y=\frac{\partial y}{\partial \delta_{1}} \Delta \delta_{1}+\frac{\partial y}{\partial \delta_{2}} \Delta \delta_{2}
$$

and

$$
\Delta z=\frac{\partial z}{\partial \delta_{1}} \Delta \delta_{1}+\frac{\partial z}{\partial \delta_{2}} \Delta \delta_{2}
$$

where $\Delta \delta_{1}$ and $\Delta \delta_{2}$ are $\Delta \delta$ of the first and the second optical gate, respectively.

$$
\begin{aligned}
& \frac{\partial y}{\partial \delta_{1}}=-\frac{D \sec ^{2} \delta_{1} \tan \delta_{2}}{\left(\tan \delta_{1}+\tan \delta_{2}\right)^{2}} \\
& \frac{\partial y}{\partial \delta_{2}}=\frac{D \tan \delta_{1} \sec ^{2} \delta_{2}}{\left(\tan \delta_{1}+\tan \delta_{2}\right)^{2}} \\
& \frac{\partial z}{\partial \delta_{1}}=\frac{D \sec ^{2} \delta_{1} \tan ^{2} \delta_{2}}{\left(\tan \delta_{1}+\tan \delta_{2}\right)^{2}}
\end{aligned}
$$

and

$$
\frac{\partial z}{\partial \delta_{2}}=\frac{D \tan ^{2} \delta_{1} \sec ^{2} \delta_{2}}{\left(\tan \delta_{1}+\tan \delta_{2}\right)^{2}}
$$

IEE PROCEEDINGS-A, Vol. 140, No. 4, JULY 1993
It is obvious from eqns $9-16$ that $N$ has to be increased as the position accuracy needs to be better for a same size of $D$.

Fig. 2 shows the intersection of the two light screens in the target area where intervals between photodiodes are considered to be constant. As indicated in this Figure, different detector positions will produce different resolutions. Fig. 7 shows simulation results of the worstcase deviations on the $y$-axis (or $z$-axis). The shotposition angle $\delta_{1}$ is fixed at $45^{\circ}$ and the activated photodiode of the second optical gate varies from number 40 to 360 on an EOTS with $D=1000 \mathrm{~mm}$ and $2 N=400$. Fig. 7 shows deviations on the $y$-axis (or $z$-axis) of less than $1 \mathrm{~mm}$ corresponding to certain photodiodes that are numbered approximately from 120 to 280. A pentagon-shaped region, which is shown in Fig. 2 and has an accuracy better than $1 \mathrm{~mm}$, also corresponds to those photodiodes.

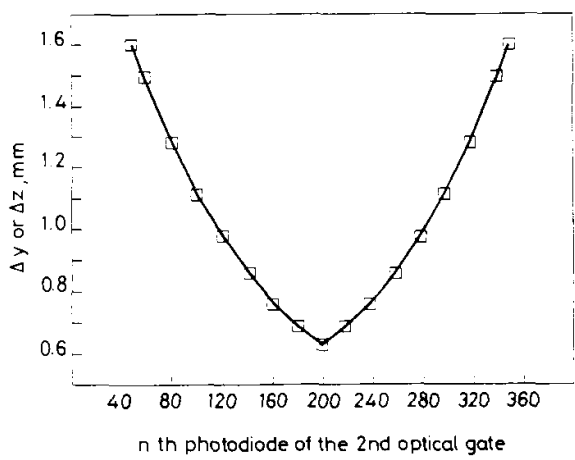

\section{Experimental results}

A prototype EOTS was used in the experiments. The main specifications of the system are listed as follows: $d=0.81 \mathrm{~mm}, w=2.5 \mathrm{~mm}, S=635 \mathrm{~mm}, D=1000 \mathrm{~mm}$, $2 N=384$ and laser output optical power $P=7.5 \mathrm{~mW}$. We hung up a paper target behind EOTS for comparison. Fig. 8 shows the $y$ and $z$ co-ordinates of nine impact points from a $0.38 \mathrm{in}$. pistol. The impact positions and the velocities were measured by EOTS. In Fig. 8, the two crosses at the bottom indicate the positions of cylindrical mirrors. Table 2 compares the results generated by EOTS with the measurement results from the paper target. The standard deviation is less than $1 \mathrm{~mm}$.

\section{Discussion and conclusions}

This paper presents a novel electro-optical target system for small calibre projectiles. Position and speed data can be generated instantaneously by the microcomputerbased control unit with the addition of appropriate software. The most accurate region of positioning, which is a pentagon-shaped area, distributes over the centre of the target area. The accuracy of position and speed measurement has been analysed in this paper. To improve the speed accuracy, we should reduce the influnce of the response time difference. To increase the distance between the two light screens, of course, is another method to improve the speed accuracy, but the position accuracy will become worse. To improve position accur- 
acy, the photodiode array which has less space between two adjacent photodiodes is suggested.

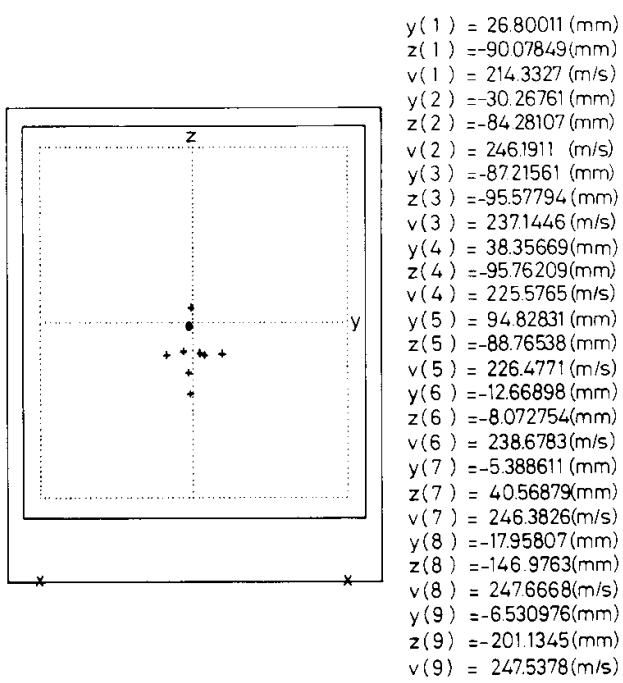

Fig. 8 The computer printout of EOTS, origin is shifted to centre of the target area

The measured speed range of EOTS is from subsonic to supersonic. A speed accuracy of better than $0.3 \%$ is accomplished. With a different design concept, EOTS need not synchronise with the firing signal as EYS. It is always ready for any advancing projectile as the power of EOTS has been turned on.

incident laser beam

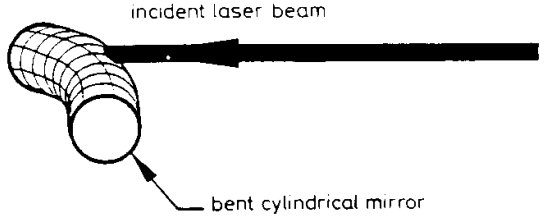

Fig. 9 Laser beam directs on a bent cylindrical mirror

If a slightly bent cylindrical mirror were used (Fig. 9), the light screen could extend a few degrees in the $x$ direction. This makes optics alignment easier and insensitive to vibrations. However, the surface quality of the cylindrical mirror is critical to the uniformity of the fanshaped beam in the $x$-direction. The nonuniformity of the fan beam in the $x$-direction will enhance the sensitivity to vibrations.

Table 2: List of results measured by EOTS and by artificial

\begin{tabular}{|c|c|c|c|c|c|c|c|}
\hline \multicolumn{2}{|c|}{$\begin{array}{l}\text { Impact } \\
\text { points }\end{array}$} & \multicolumn{2}{|c|}{$\begin{array}{l}\text { Distance } \\
\text { measured by }\end{array}$} & \multicolumn{2}{|c|}{$\begin{array}{l}\text { Impact } \\
\text { points }\end{array}$} & \multicolumn{2}{|c|}{$\begin{array}{l}\text { Distance } \\
\text { measured by }\end{array}$} \\
\hline from & to & EOTS & artificial & from & to & EOTS & artificial \\
\hline & & $\mathrm{mm}$ & $\mathrm{mm}$ & & & $\mathrm{mm}$ & $\mathrm{mm}$ \\
\hline I & 2 & 57.36 & 57.5 & 3 & 7 & 158.85 & 159.5 \\
\hline & 3 & 114.15 & 115.0 & 3 & 8 & 86.25 & 86.5 \\
\hline & 4 & 12.88 & 13.0 & 3 & 9 & 132.86 & 133.5 \\
\hline | & 5 & 68.04 & 69.0 & 4 & 5 & 56.90 & 58.0 \\
\hline & 6 & 91.01 & 92.0 & 4 & 6 & 101.46 & 102.0 \\
\hline 1 & 7 & 134.56 & 135.0 & 4 & 7 & 143.18 & 144.0 \\
\hline 1 & 8 & 72.40 & 72.5 & 4 & 8 & 76.13 & 75.5 \\
\hline$i$ & 9 & 115.94 & 117.0 & 4 & 9 & 114.53 & 114.0 \\
\hline 2 & 3 & 58.06 & 58.0 & 5 & 6 & 134.42 & 135.0 \\
\hline 2 & 4 & 69.58 & 69.0 & 5 & 7 & 163.62 & 163.0 \\
\hline 2 & 5 & 125.18 & 125.5 & 5 & 8 & 126.93 & 127.5 \\
\hline 2 & 6 & 78.22 & 79.0 & 5 & 9 & 151.32 & 152.5 \\
\hline 2 & 7 & 127.30 & 128.0 & 6 & 7 & 49.18 & 49.0 \\
\hline 2 & 8 & 63.90 & 64.0 & 6 & 8 & 139.01 & 140.0 \\
\hline 2 & 9 & 119.24 & 120.0 & 6 & 9 & 193.16 & 195.0 \\
\hline 3 & 4 & 125.58 & 124.5 & 7 & 8 & 187.97 & 188.5 \\
\hline 3 & 5 & 182.18 & 182.5 & 7 & 9 & 241.70 & 243.0 \\
\hline 3 & 6 & 114.96 & 115.5 & 8 & 9 & 55.34 & 56.0 \\
\hline
\end{tabular}

Comparing EOTS with EYS and other conventional methods, EOTs has the following advantages:

(a) It can measure position and speed precisely and simultaneously.

(b) Its optical system is simple and easy to set up.

(c) It is insensitive to vibrations if a bent cylindrical mirror is used.

(d) Its speed range covers subsonic to supersonic.

\section{References}

I BETTERMANN, P., and MAYER, F.: 'Handbook on weaponry' (Rheinmetall GmbH, Düsseldorf, 1982)

2 FARRAR, C.L., and LEEMING, D.W.: 'Military ballistics' (Brassey's Publishers Limited, 1983)

3 CRITTENDEN, E.C., KING, R.A., and ANDREWS, T.C.: 'Target measurement system for precise projectile location'. US Patent No. 3727069, 1973

4 BAILEY, T.B., and BATES, J.: 'Electro-optical projectile analyzer' US Patent No. 4272189,1981

5 DECK, L.L.: 'An optical device for rapid measurement of the speed dispersion, attack angle and shock wave of high velocity small caliber projectiles'. Proceedings of 10 th International Symposium on Ballisprojectiles . Proceeding

6 LU, S.T., YU, A.T., and CHOU, C.: 'Electro-optics target for position and speed measurement', Proc. SPIE, 1988, 981, pp. 250-254 7 TUINENGA, P.W.: 'SPICE a guide to circuit simulation and analysis using PSpice' (Prentice-Hall, 1988)

8 HARTWIG, R: 'Accuracy of velocity measurement of projectiles with fins and tracers by means of sky-screens', J. Ballistics, 1986, 9, (3), pp. 2299-2310 